\title{
Supplementation of Rhodomyrtus tomentosa fruit polyphenols improved dextran sulfate induced colitis in mice by regulating gut microbiota
}

\author{
Jianing Zhang ${ }^{1}$, Shuyuan Chai ${ }^{1}$, Xi Jia ${ }^{1}$, Yujin $\mathrm{Han}^{1}$, Yufei Liu ${ }^{1}$, Xiaohong $\mathrm{He}^{2}$, Yanmei $\mathrm{Li}^{3}$, and Fahu Yuan ${ }^{1, *}$ \\ ${ }^{1}$ School of medicine, Jianghan University, Wuhan, Hubei, China \\ ${ }^{2}$ Enshi Center for Disease Control and Prevention, Enshi Tujia and Miao Autonomous Prefecture, Hubei, China \\ ${ }^{3}$ Hubei Key Laboratory of Applied Toxicology, Wuhan, Hubei, China
}

\begin{abstract}
To investigate the therapeutic effect of polyphenol from the fruits of Rhodomyrtus tomentosa (RTFP) on experimental colitis induced by dextran sulphate sodium(DSS) in mice. A total of $24 \mathrm{Babl} / \mathrm{c}$ mice were randomly divided into normal control group, colitis model group and DSS+RTFP intervention group, respectively. The intestinal disease activity index (DAI), pathological histology of colon were investigated, and the changes of gut microbiota in mice were evaluated by 16S rRNA sequencing. Compared with the DSS-induced colitis group, RTFP showed intestinal anti-inflammatory effects, the DAI score was significantly decreased, RTFP improved colitis induced weight loss, fecal imformation and blood in the stool. RTFP relieved the phenomenon of shortening colon length, shortening intestinal wall, and splenomegaly. RTFP intervention inhibited the increase of bacteroides abundance caused by DSS, and remodeled the diversity of gut microbiota. Taken together, RTFP could effectively intervene in experimental colitis induced by DSS in mice, which may be related to the modulating gut microbiota and intestinal cell immune balance.
\end{abstract}

\section{Introduction}

Inflammatory bowel disease(IBD), is based on the intestinal inflammation and damage of the intestinal epithelial pathological features of chronic recurrent gastrointestinal tract disease. IBD include ulcerative colitis(UC) and Crohn disease(CD), UC is one of a group of chronic diseases of inflammatory bowel disease [1]. Patients with IBD is not only the serious influence the quality of life, chronic inflammation can easily induce colorectal tumors, caused a great economic burden to society. The traditional treatment of IBD include glucocorticoid and immune inhibitors, but these treatments can't significantly shorten the duration of IBD, and long-term use of the above drugs can bring difficult to overcome the adverse reaction of patients. Therefore, it is urgent to find new drugs or intervention measures to safely and effectively inhibit the occurrence of enteritis and reduce the incidence of enteritis.

At present, IBD is believed to be caused by the interaction of multiple factors such as heredity, immunity and environment. The combination of genetic environment and changes in gut flora stimulates an abnormal immune response that leads to chronic intestinal inflammation. In addition, epidemiological and clinical evidence suggests that IBD is associated with a number of environmental factors in which diet plays an important role in intestinal function and immune activity, the microbial metabolism of food components in the intestinal tract can affect the intestinal epithelial barrier and mucosal immune system. Dietary composition plays an important role in intestinal barrier function of intestinal flora, immune system and epigenetics, which is expected to provide theoretical basis for future exploration of IBD dietary interventions[2].

The imbalance of gut microbiota and the destruction of host-microbial symbiosis may be the decisive events in the development of IBD[3]. With the development of molecular biology and metagenomics, more and more studies have shown that the gut microbiota of IBD patients has undergone significant changes, and the intestinal flora plays an important role in the pathogenesis of IBD. Compared with healthy individuals, IBD patients had an increase in inflammatory bacteria and a decrease in anti-inflammatory bacteria, as well as a decrease in the diversity of intestinal microbiota and a decrease in the abundance of firmicutes, thereby changing the diversity and composition of intestinal microbiota. The increase of pathogenic bacteria capable of adhesion to intestinal epithelium, such as invasive Escherichia coli, can affect intestinal permeability and induce inflammatory response by regulating the expression of inflammatory genes, thus leading to the occurrence of intestinal inflammation[4].

Polyphenols have anti-inflammatory, antioxidant, anti-obesity, anti-angiogenesis, anti-cancer, anti-diabetes and immunomodulatory effects[5]. A large number of studies have shown that dietary polyphenols have a

* Corresponding author: yuanfh@jhun.edu.cn 
strong inhibitory effect on colitis and colon cancer. Polyphenols can protect the integrity of the intestinal mucosa, maintain the intestinal epithelial barrier function, restore the intestinal mucosal immune regulation function, and improve the intestinal microecology so as to regulate and play its anti-inflammatory effect.

Based on the above analysis, we hypothesized that the regulation of intestinal epithelial related immune microenvironment by remodeling intestinal microecology, thereby inhibiting the progression of IBD, is an important mechanism of dietary intervention for IBD.

\section{Material and method}

\subsection{Plant materials and preparation of polyphenols extract from the fruit of Rhodomyrtus tomentosa}

Fresh Rhodomyrtus tomentosa fruit was collected from a farm from Shaoguan, Guangdong, China. After removing the impurities, dry them in a $50^{\circ} \mathrm{C}$ drying oven. The dried sample is ground into a fine powder by an ultrafine pulverizer. Then ethanol $(90 \%, 1: 4 / \mathrm{wt}: \mathrm{vol})$ was used for ultrasonic assisted extraction on the agitator at $50{ }^{\circ} \mathrm{C}$ for $3 \mathrm{~h}$. Then the supernatant was collected by centrifugation at $1000 \mathrm{~g}$ for $10 \mathrm{~min}$. Then, as mentioned earlier, the residue is extracted with ethanol twice more.Finally, the three supernatants were mixed and concentrated in a vacuum concentrator at $50{ }^{\circ} \mathrm{C}$ until no distillate was obtained. The polyphenols extract from the fruit of Rhodomyrtus tomentosa(RTFP) was collected and stored at $4{ }^{\circ} \mathrm{C}$ for use.

\subsection{Ethics statement and animal experimental design}

Male BALB/ C mice (6-8 weeks old) were purchased from the Experimental Animal Center of Hubei Provincial Center for Disease Control and Prevention. The mice were fed in SPF level animal laboratory under the control of $(24 \pm 1)^{\circ} \mathrm{C}$ and $(50 \pm 5) \%$ humidity for 12 hour light and dark cycle. Before the experiment, the mice were fed with water and food at will and acclimated for a week. All animal procedures in this study were in accordance with the guidelines for the care and use of laboratory animals issued by the Academic Ethics Committee of Jianghan University and approved by the Jianghan University Animal Protection and Use Committee. Mice were randomly divided into CON group, DSS group and RTFP group, with 8 mice in each group. Dextran Sodium Sulfate (DSS) is a polyionic derivative of Dextran. DSS modeling method is simple and inexpensive, and its clinical, pathological and pathological features are very similar to human. On day 1-14, The RTFP group was given $500 \mathrm{mg} / \mathrm{kg}$ RTFP and gavage once a day. The CON and DSS mice were given the same volume of distilled water. On day 15-21, DSS and RTFP mice were given 3\% DSS solution (diluted with distilled water), but during this period, the CON group and the RTFP group continued to receive distilled water and RTFP gavage.

Weight and key parameters of disease activity index (DAI), such as diarrhea and blood stool, were monitored every two days throughout the study period. DAI scores are calculated according to the DAI scoring system. DAI criteria are presented in TABLE 1 . On day 22, all mice were sacrificed and serum and colon were collected. The length of the colon is determined with a ruler. A part of the colon specimens were histologically analyzed with $4 \%$ paraformaldehyde solution fixation, the other part was rapidly frozen in liquid nitrogen and then stored at $80^{\circ} \mathrm{C}$ for biochemical index analysis.

Table 1. The standard of disease activity index (DAI)

\begin{tabular}{|c|c|c|c|}
\hline Score & $\begin{array}{c}\text { Weight } \\
\text { loss (\%) }\end{array}$ & $\begin{array}{c}\text { Stool } \\
\text { consistency }\end{array}$ & $\begin{array}{c}\text { Occult } \\
\text { /gross bleeding }\end{array}$ \\
\hline 0 & $<1$ & Normal stools & None \\
\hline 1 & $1-5$ & $\begin{array}{c}\text { Soft pellets not } \\
\text { adhering to the } \\
\text { anus }\end{array}$ & $\begin{array}{c}\text { Small spots of blood stool } \\
\text { dry analregion }\end{array}$ \\
\hline 2 & $5-10$ & $\begin{array}{c}\text { Very soft pellets } \\
\text { adhering to the } \\
\text { anus }\end{array}$ & $\begin{array}{c}\text { Large spots of blood in } \\
\text { stool bloodappears } \\
\text { through the anal orifice }\end{array}$ \\
\hline 3 & $10-15$ & $\begin{array}{c}\text { Liquid stool in } \\
\text { long streams wet } \\
\text { anus }\end{array}$ & $\begin{array}{c}\text { Deep red stool blood } \\
\text { spreads largelyaround the } \\
\text { anus }\end{array}$ \\
\hline 4 & $>15$ & Gross bleeding & Diarrhea \\
\hline
\end{tabular}

\subsection{Histology}

Colon tissue was fixed with $4 \%$ paraformaldehyde solution, dehydrated with ethanol gradient, and prepared $4 \mathrm{~m}$ thick continuous sections after paraffin embedding. The sections were dewaxed, stained with hematoxylin and eosin(H\&E) staining, and sealed with neutral resin. Pathological grading of colon tissue was evaluated.

\subsection{Sequencing analysis of gut microbiota based on the 16S rRNA}

Omega fecal DNA extraction kit was used to extract DNA from feces. The extraction quality of DNA was detected by $0.8 \%$ agar-gel electrophoresis and quantified by ultraviolet spectrophotometer. Using the extracted DNA as the template, Barcode specific primer was used to carry out PCR amplification of $16 \mathrm{~S}$ rRNA gene in the v3-v4 region, and the cycle number was controlled to be 3 . The PCR products were quantified by $2 \%$ gels electrophoresis, and the target fragments were recovered.

Illumina's TruSeq Nano DNA LT Library Prep Kit was used to prepare the sequencing Library. After quantification and Library detection were qualified, Illumina MiSeq platform was used for sequencing. The original data were splice and filtered to obtain high quality target sequences. $97 \%$ sequence similarity was used as the Operational Taxonomic Unit (OTU) classification threshold. Species annotation was performed on OTUs according to the clustering results, and specaccum species accumulation curve analysis, 
Alpha diversity analysis and Beta diversity analysis were conducted after obtaining the species information.

\subsection{Statistical analysis}

SPSS 21.0 statistical software was used for statistical analysis of conventional data, Measurement data presented as mean \pm standard deviation. For multiple comparisons, one-way analysis of variance (ANOVA) with Newman-Keuls' s post-hoc test was used. A value of $P<0.05$ was regarded as significant.

\section{Results}

\subsection{Body weight}

To determine whether RTFP attenuates DSS-induced colon inflammation, mice were given 3\% DSS of drinking water for 7 days. The DSS group showed a continuous 7 days of weight loss(Fig. 1). In contrast, mice given DSS+RTFP showed higher body weight than mice given DSS alone after DSS treatment. These results suggest that DSS induced weight loss recovery is more effective in RTFP-treated mice.

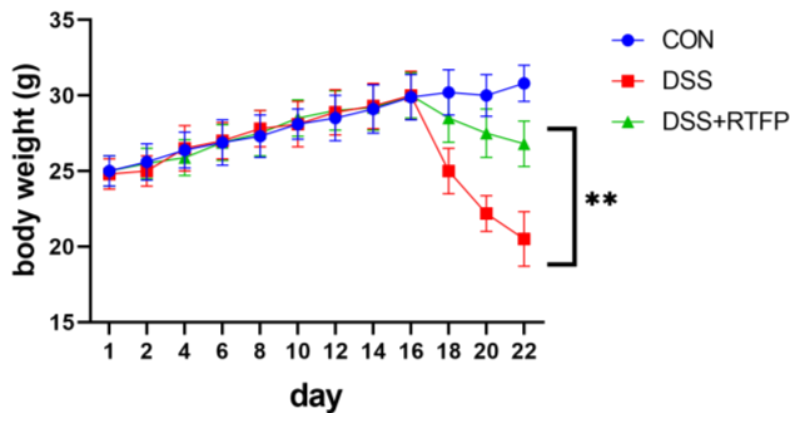

Fig. 1. Effect of RTFP on the body weight of DSS-treated mice. $* * P<0.01$.

\subsection{DAI and colon length}

Disease activity index (DAI) is used to judge the severity of inflammation in mice according to the weight loss, fecal viscosity and fecal blood during modeling. DAI score of mice in each group during modeling is shown in Figure 2. From day 18, mice in the DSS group and the RTFP+DSS group showed a significant trend of weight loss, accompanied by loose stools in some mice. Compared with the normal group, mice that drank DSS had less food and water, and their hair was no longer supple. On the 20th day, some mice in the DSS group and PMF+DSS group had blood in their stool, and most of the mice had diarrhea. Compared with the normal group, the DSS mice began to have slow movement, no light in their eyes, and the diet and drinking water continued to decline. On the 7th day of modeling, all the mice in the DSS group had blood in their stool, among which 6 mice showed massive hemorrhage and the degree of diarrhea was aggravated. Moreover, 3 mice were no longer active and their hair lost luster. The degree of stool blood and diarrhea in RTFP+DSS group was less than that in DSS group, and all mice were in low frequency activity state. The experimental results showed that RTFP could reduce the degree of stool blood in colitis model mice, relieve diarrhea and slow down the trend of weight loss in mice.

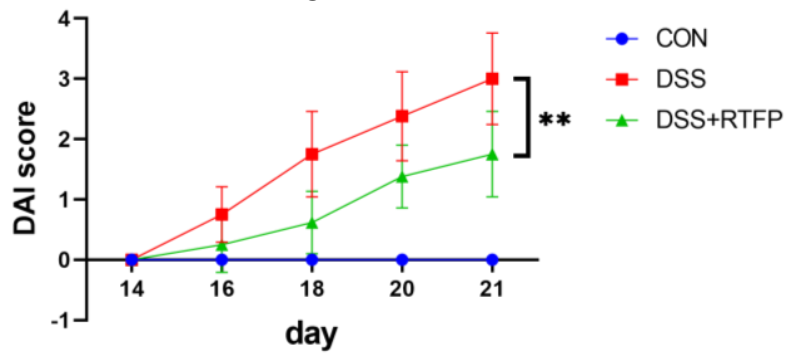

Fig. 2. Effect of RTFP on DAI score of colitis mice. ${ }^{* *} P<0.01$.

In the model of DSS-induced colitis in mice, colon morphology and length are one of the important indicators to evaluate the severity of inflammation in colitis mice, intestinal swelling, bleeding, ulcer, intestinal wall thickening and other pathological reactions will occur in colitis mice. As shown in Figure 3, the intestines of mice that had DSS were significantly shorter than those of the normal control group $(P<0.01)$. The mean length was $9.73 \mathrm{~cm}$ in CON group, $5.31 \mathrm{~cm}$ in DSS group, and $7.45 \mathrm{~cm}$ in RTFP+DSS group, respectively. The intestinal wall of mice in DSS group has obvious dark red petechiae, and intestinal wall become fragile, because a large amount of bleeding, the excrement in the cecum and colon contents present black, colon waste because of edema, compared with normal group cannot be forming, and RTFP + DSS mice compared with DSS group, the phenomenon of bleeding from the walls of her intestines eased, cecum contents color becomes shallow, colon feces to forming state close to normal mice.
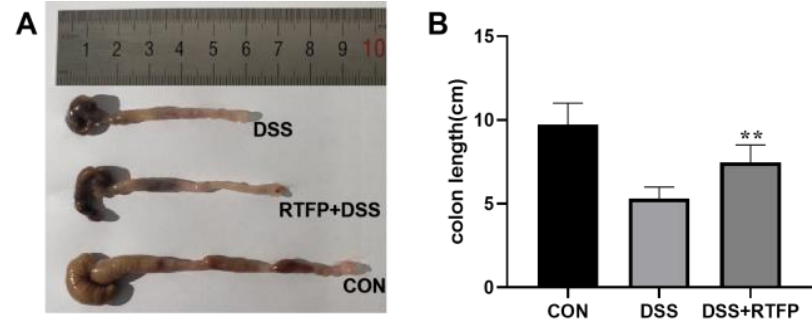

Fig. 3. Effects of RTFP on colonic length in colitis mice. $* * \mathrm{P}<0.01$

\subsection{Histopathology}

The H\&E staining of the colon section showed that LRE improved histopathological lesions caused by DSS, including goblet cell reduction, crypt loss, mucosal ulcers, inflammatory infiltration, and necrosis (Fig. 4). 
CON

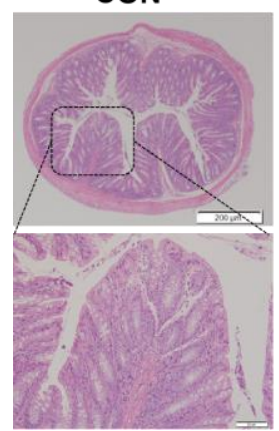

DSS

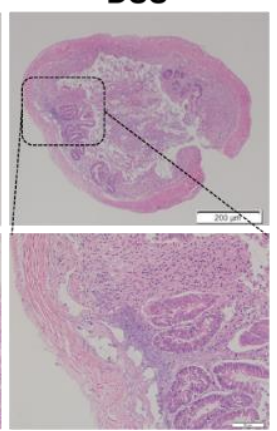

DSS+RTFP

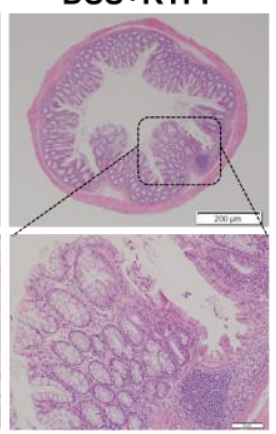

Fig. 4. Colonic histologic

\subsection{OTUs and diversity estimates for fecal microbiota}

To analyze gut microbiota, fecal samples were collected and they were analyzed using sequencing. Differences in the microbiota among the groups were compared at the phylum level (Fig. 5). DSS administration greatly increased the levels of Bacteroidetes by $35.8 \%$, and decreased the levels of Firmicutes by $30.2 \%$ compared to the CON group. Correspondingly, the ratio of Bacteroidetes to Firmicutes in the gut microbiota was higher for the DSS group compared to the CON group. However, following RTFP treatment, the proportions of Bacteroidetes and Firmicutes returned to normal levels. Moreover, the ratio of Bacteroidetes to Firmicutes decreased following RTFP supplementation. In contrast, the levels of Proteobacteria and Deferribacteres did not significantly different among the three groups.

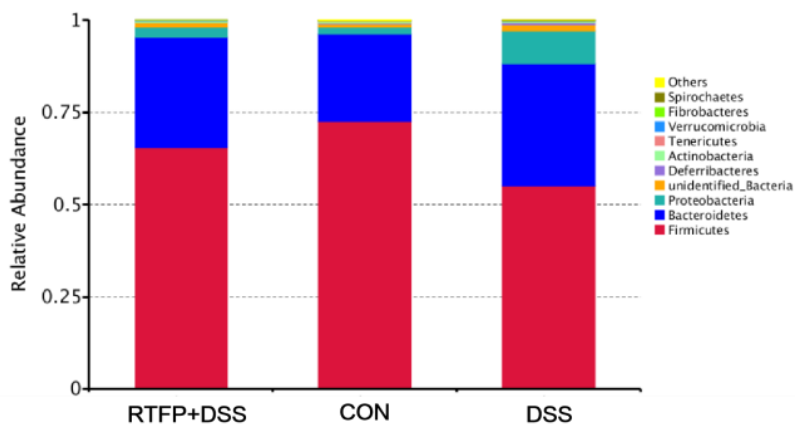

Fig. 5. OTUs and diversity estimates for fecal microbiota

\section{Conclusion}

This study has shown that RTFP can improve the colitis induced weight loss, fecal imformation and blood in the stool, as well as relieve the shortened colon length, bleeding, intestinal wall fragility, spleen enlargement and other phenomena. However, when RTFP was administered prior to the induction of colitis by DSS, microbial dysbiosis was reduced. These results increase our understanding of the important role that gut microbacteria have in maintaining intestinal homeostasis, and they also support the natural therapeutic potential of RTFP for modulating dysbiosis in IBD.

\section{References}

1. Liu Jimmy Z., van Sommeren Suzanne., Huang Hailiang., Ng Siew C., Alberts Rudi., Takahashi Atsushi., Ripke Stephan., Lee James C., Jostins Luke., Shah Tejas., Abedian Shifteh., Cheon Jae Hee., Cho Judy., Dayani Naser E., Franke Lude., Fuyuno Yuta., Hart Ailsa., Juyal Ramesh C., Juyal Garima., Kim Won Ho., Morris Andrew P., Poustchi Hossein., Newman William G., Midha Vandana., Orchard Timothy R., Vahedi Homayon., Sood Ajit., Sung Joseph Y., Malekzadeh Reza., Westra HarmJan., Yamazaki Keiko., Yang Suk-Kyun., International Multiple Sclerosis Genetics Consortium., International IBD Genetics Consortium., Barrett Jeffrey C., Alizadeh Behrooz Z., Parkes Miles., Bk Thelma., Daly Mark J., Kubo Michiaki., Anderson Carl A., Weersma Rinse K.(2015). Association analyses identify 38 susceptibility loci for inflammatory bowel disease and highlight shared genetic risk across populations. Nat. Genet., 47(9), 979-986.

2. Kaplan Gilaad G.(2015). The global burden of IBD: from 2015 to 2025. Nat Rev Gastroenterol Hepatol, 12(12), 720-7.

3. Bellaguarda Emanuelle., Chang Eugene B.(2015). IBD and the gut microbiota--from bench to personalized medicine. Curr Gastroenterol Rep, 17(4), 15.

4. Ananthakrishnan Ashwin N.(2015). Epidemiology and risk factors for IBD. Nat Rev Gastroenterol Hepatol, 12(4), 205-17.

5. Salucci Sara., Falcieri Elisabetta.(2020). Polyphenols and their potential role in preventing skeletal muscle atrophy. Nutr Res, 74(undefined), 10-22. 